\title{
ON THE UNIFORM DISTRIBUTION IN POSITIVE CHARACTERISTIC
}

\section{ZHIYONG ZHENG ${ }^{1}$, ZIWEI HONG ${ }^{2}$ and MAN CHEN ${ }^{3}$}

${ }^{1}$ School of Mathematics

Renmin University of China

Beijing

P. R. China

e-mail: zhengzy@ruc.edu.cn

${ }^{2}$ School of Mathematics and Systems Science

Beihang University

Beijing

P. R. China

e-mail: hongziwei@live.com

${ }^{3}$ Department of Mathematics

South China University of Technology

Guangzhou

P. R. China

e-mail: 13798043026@163.com 


\begin{abstract}
Uniform distribution is an important subject in classical Diophantine approximation. There is a close connection between the distribution of real numbers and the estimation of exponential sums via Weyl's criteria. Carlitz gave a definition of uniform distribution in positive characteristic in an elementary way (see [11]), however, we are going to find a geometrical description. In this paper, we present a precise analogue to Weyl's criteria in the case of positive characteristic by using Haar measure. As an application, we show that the uniformly distributed modulo 1 for linear forms and for polynomial functions. In particular, we prove the set $\{m \theta\}$ in the Laurent series field is uniformly distributed modulo 1 , where $m$ extends over all the polynomials and $\theta$ is a fixed irrational function.
\end{abstract}

\title{
1. Introduction
}

Let $\mathbb{F}_{q}$ be a finite field with $q$ elements of characteristic $p$, $K=\mathbb{F}_{q}[T]$ be the polynomial ring, $k=\mathbb{F}_{q}(T)$ be the rational function field, and $k_{\infty}=\mathbb{F}_{q}\left(\left(\frac{1}{T}\right)\right)$ be the formal Laurent series field. Let $v$ be the normalized exponential valuation of $k_{\infty}$ with $v\left(\frac{1}{T}\right)=1$ and $v(0)=\infty$. If $\alpha$ is an element in $k_{\infty}$, then $\alpha$ can be uniquely expressed as a Laurent series as follows:

$$
\alpha=\sum_{i=n}^{+\infty} a_{i}\left(\frac{1}{T}\right)^{i}, \quad n \in \mathbb{Z}, a_{i} \in \mathbb{F}_{q} \text {, and } a_{n} \neq 0
$$

where $v(\alpha)=n$. We define the square bracket function $[\alpha]$ by

$$
[\alpha]=\sum_{i=n}^{0} a_{i}\left(\frac{1}{T}\right)^{i} \text {, if } n \leq 0 \text {, and }[\alpha]=0 \text {, if } n>0 \text {, }
$$

which is called the "integral part" of $\alpha$ as usual. We see that $[\alpha] \in K$, $[\alpha+\beta]=[\alpha]+[\beta],[a \alpha]=a[\alpha]$ for all $a \in \mathbb{F}_{q}^{*}$, and $v(\alpha-[\alpha]) \geq 1$. In fact, there is a unique polynomial $A=[\alpha]$, such that $v(\alpha-A) \geq 1$. We write 
$\langle\alpha\rangle=\alpha-[\alpha]$, which is called the "fractional part" of $\alpha$. It is easily seen that $\langle\alpha+\beta\rangle=\langle\alpha\rangle+\langle\beta\rangle,\langle a \alpha\rangle=a\langle\alpha\rangle$ for all $a \in \mathbb{F}_{q}$, and $\langle\alpha+A\rangle=\langle\alpha\rangle$ for all $A \in K$, in particular, we have $\langle\alpha\rangle=0$ if and only if $\alpha \in K$. The absolute value functions $|\alpha|$ and $\|\alpha\|$ in $k_{\infty}$ are given by

$$
|\alpha|=q^{-v(\alpha)} \text { and }\|\alpha\|=|\langle\alpha\rangle|
$$

It is worth to keep in mind that $|0|=0,|a|=1$ for all $a \in \mathbb{F}_{q}^{*}$, and $|\alpha \beta|=|\alpha| \cdot|\beta|$ for $\alpha, \beta \in k_{\infty}$. For the double absolute function, we have $\|\alpha+A\|=\|\alpha\|$ for all $A \in K,\|\alpha \alpha\|=\|\alpha\|$ for all $a \in \mathbb{F}_{q}^{*}, 0 \leq\|\alpha\| \leq \frac{1}{q}$ for all $\alpha \in k_{\infty}$, and $\|\alpha\|=0$ if and only if $\alpha \in K$. In particular, we have

$$
\|\alpha+\beta\| \leq \max \{\|\alpha\|,\|\beta\|\} \text {, and }\|\alpha\|=\inf _{A \in K}|\alpha-A| .
$$

Thus, $\|\alpha\|$ is the smallest distance from $\alpha$ to any element of $K$, and $[\alpha]$ is the nearest polynomial to $\alpha$.

The valuation ring $\mathfrak{P}_{0}$ and the valuation ideal $\mathfrak{P}$ of $k_{\infty}$ are given by

$$
\mathfrak{P}_{0}=\left\{\alpha \in k_{\infty}:|\alpha| \leq 1\right\} \text {, and } \mathfrak{P}=\left\{\alpha \in k_{\infty}:|\alpha|<1\right\}
$$

If $n$ is an integer, the fractional ideal $\mathfrak{P}_{n}$ is given by

$$
\mathfrak{P}_{n}=\left(\frac{1}{T}\right)^{n} \mathfrak{P}_{0}=\left\{\alpha \in k_{\infty}:|\alpha| \leq q^{-n}\right\}
$$

Obviously, $\mathfrak{P}_{1}=\mathfrak{P}$ and

$$
\cdots \supset \mathfrak{P}_{-2} \supset \mathfrak{P}_{-1} \supset \mathfrak{P}_{0} \supset \mathfrak{P}_{1} \supset \mathfrak{P}_{2} \supset \cdots
$$

The collection $\left\{\mathfrak{P}_{n}\right\}_{n \in \mathbb{Z}}$ is a fundamental system of neighbuorhoods of 0 . If $\alpha \in k_{\infty}$, we denote a ball by $\alpha+\mathfrak{P}_{n}$

$$
\alpha+\mathfrak{P}_{n}=\left\{x \in k_{\infty}:|x-\alpha| \leq q^{-n}\right\},
$$

which is usually said to be a ball of center $\alpha$ and radius $q^{-n}$. 
Let $k_{\infty}^{+}$be the additive group of $k_{\infty}$. Since $k_{\infty}^{+}$is a local compact topological group, there exists a unique Haar measure, up to a positive multiplicative constant. Define by $\mu$ the Haar measure on $k_{\infty}^{+}$normalized to have total mass 1 on $\mathfrak{P}$, we thus have

$$
\mu\left(\mathfrak{P}_{n}\right)=q^{1-n} \text {, and } \mu\left(\alpha+\mathfrak{P}_{n}\right)=q^{1-n}, \quad n \in \mathbb{Z}, \alpha \in k_{\infty} .
$$

We write $d x=d \mu(x)$, and denote by $L^{1}\left(k_{\infty}\right)$ the set of all complex valued measurable functions on $k_{\infty}$ such that

$$
\int_{k_{\infty}}|f(x)| d x<\infty
$$

Let $\hat{k}_{\infty}^{+}$be the dual group of $k_{\infty}^{+}$, which is the set of all continuous group homomorphism from $k_{\infty}^{+}$to the circle group $\mathbb{T}=\{z \in \mathbb{C}:|z|=1\}$. Every element $\psi$ in $\hat{k}_{\infty}^{+}$is said to be an additive character of $k_{\infty}$. Let $\psi_{0}$ be the principal additive character. For each additive character $\psi \neq \psi_{0}$, there exists an integer $n$ such that $\psi$ is trivial on $\mathfrak{P}_{n}$. Let

$$
n(\psi):=\min \left\{n: \psi(x)=1 \text { for evey } x \in \mathfrak{P}_{n}\right\},
$$

which is called the conductor of $\psi$ (see [4], (2.6)). We set $n\left(\psi_{0}\right)=\infty$. If $\alpha \in k_{\infty}$, and $\psi \in \hat{k}_{\infty}^{+}$, we define $\psi_{\alpha}(x)=\psi(\alpha x)$ for all $x \in k_{\infty}$. Clearly, $\psi_{\alpha}$ is again an additive character of $k_{\infty}$, and the conductor of $\psi_{\alpha}$ is given by (see [4], (2.14))

$$
n\left(\psi_{\alpha}\right)=n(\psi)-v(\alpha)
$$

In the preceding papers $[9,10]$, we showed that a few basic results on the simultaneous Diophantine approximation in $k_{\infty}$. In particular, we showed in [10] that the set $\{\langle m \theta\rangle\}_{m \in K}$ are everywhere dense in the valuation ideal $\mathfrak{P}$. For a general background to material on Diophantine 
approximation in characteristic zero and in positive characteristic, we refer the reader to [1, 2, 3, 7] as well as the survey papers [5, 8]. In this paper, we define and describe the uniform distribution modulo 1 for $k_{\infty}$, and present a precise analogue to Weyl's criterion in the case of positive characteristic. In particular, we prove the set $\{m \theta\}_{m \in K}$ is uniformly distributed modulo 1 .

To state our definitions and results, let $z^{(m)}=\left(z_{m 1}, z_{m 2}, \ldots, z_{m s}\right)$ $(1 \leq m \leq M)$ be $M s$-dimensional vectors in $\mathfrak{P}^{s}$, let $\alpha=\left(\alpha_{1}, \alpha_{2}, \ldots, \alpha_{s}\right)$ $\in \mathfrak{P}^{s}$ and $r=\left(r_{1}, \ldots, r_{s}\right) \in \mathbb{Z}^{s}$ with $r_{j} \geq 1$. We denote by $F_{M}(\alpha, r)$ a counting numbers function as follows:

$$
F_{M}(\alpha, r)=\#\left\{z^{(m)}: 1 \leq m \leq M \text {, and } z_{m j} \in \alpha_{j}+\mathfrak{P}_{r_{j}} \text { for all } 1 \leq j \leq s\right\} .
$$

The discrepancy of the sequence $\left\{z^{(m)}\right\}_{1 \leq m \leq M}$ are defined by

$$
\begin{aligned}
D_{M}= & \sup _{\substack{\alpha \in \mathfrak{P}^{s} \\
r \in \mathbb{Z}^{s}, r_{j} \geq 1}}\left|M^{-1} F_{M}(\alpha, r)-\prod_{j=1}^{s} \mu\left(\alpha_{j}+\mathfrak{P}_{r_{j}}\right)\right| \\
= & \sup _{\substack{\alpha \in \mathfrak{P}^{s} \\
r \in \mathbb{Z}^{s}, r_{j} \geq 1}}\left|M^{-1} F_{M}(\alpha, r)-q^{s-\sum_{j=1}^{s} r_{j}}\right|,
\end{aligned}
$$

Definition 1.1. If $\lim _{M \rightarrow \infty} D_{M}=0$, we call the sequence $\left\{z^{(m)}\right\}_{1 \leq m<\infty}$ in $\mathfrak{P}^{s}$ uniformly distributed. Suppose that $z^{(m)}(1 \leq m \leq M)$ are $M$ vectors in $k_{\infty}^{s}$, not necessarily restricted to lie in $\mathfrak{P}^{s}$. Let $\left\langle z^{(m)}\right\rangle=\left(\left\langle z_{m 1}\right\rangle,\left\langle z_{m 2}\right\rangle, \ldots,\left\langle z_{m s}\right\rangle\right)$ be the fractional parts vector of $z^{(m)}$. If $\left\langle z^{(m)}\right\rangle(1 \leq m<\infty)$ is uniformly distributed in $\mathfrak{P}^{s}$, then we call the sequence $\left\{z^{(m)}\right\}_{1 \leq m<\infty}$ uniformly distributed modulo 1 . 
The main results of this paper are the following theorems.

Theorem 1.1 (Weyl's criteria). Let $z^{(m)}(1 \leq m<\infty)$ be a sequence of vectors in $\mathfrak{P}^{s}$. Then the following statements are equivalent:

(i) $z^{(m)}(1 \leq m<\infty)$ is uniformly distributed in $\mathfrak{P}^{s}$.

(ii) For all real or complex valued Haar-integrable functions $f(z)=f\left(z_{1}, z_{2}, \ldots, z_{s}\right)$ on $\mathfrak{P}^{s}$, we have

$$
\lim _{M \rightarrow \infty} M^{-1} \sum_{1 \leq m \leq M} f\left(z^{(m)}\right)=\int_{\mathfrak{P}} \cdots \int_{\mathfrak{P}} f(z) d z_{1} d z_{2} \cdots d z_{s}
$$

(iii) Let $\psi$ be an additive character of $k_{\infty}$ with the conductor $n(\psi)=2$.

Then we have

$$
\lim _{M \rightarrow \infty} M^{-1} \sum_{1 \leq m \leq M} \psi\left(A \cdot z^{(m)}\right)=0
$$

for all nonzero vectors $A=\left(A_{1}, A_{2}, \ldots, A_{s}\right) \in K^{s}$, where $A \cdot z^{(m)}$ is the inner product of vectors $A$ and $z^{(m)}$ given by

$$
x \cdot y=x_{1} y_{1}+x_{2} y_{2}+\cdots+x_{s} y_{s} \text {, where } x=\left(x_{1}, \ldots, x_{s}\right), y=\left(y_{1}, \ldots, y_{s}\right) .
$$

Corollary 1.1. Let $z^{(m)}(1 \leq m<\infty)$ be a sequence of vectors in $k_{\infty}^{s}$, not necessarily restricted to lie in $\mathfrak{P}^{s}$. Suppose that $\psi$ is an additive character on $k_{\infty}$ such that $n(\psi)=2$ and $\psi(\alpha+A)=\psi(\alpha)$ for all $\alpha \in k_{\infty}$ and $A \in K$. The necessary and sufficient conditions that $\left\{z^{(m)}\right\}$ be uniformly distributed modulo 1 is that

$$
\lim _{M \rightarrow \infty} M^{-1} \sum_{1 \leq m \leq M} \psi\left(A \cdot z^{(m)}\right)=0
$$

for all nonzero vectors $A=\left(A_{1}, \ldots, A_{s}\right) \in K^{s}$. 
In Section 3 below, we shall introduce an additive character $\psi$ such that $n(\psi)=2$ and $\psi(\alpha+A)=\psi(\alpha)$ for all $\alpha \in k_{\infty}, A \in K$, of which may be regarded as an analogue to the exponential function $e^{2 \pi i x}$ in the complex number field. Therefore, the summation on the left-hand side of (1.14) may be regarded as the exponential sums in $k_{\infty}$.

To apply the above Weyl's criteria, we next show that some of classical examples of uniformly distributed modulo 1. First, we consider the simplest case as follows.

Theorem 1.2. Suppose that $\theta \in k_{\infty}$ and $\theta \notin k$. Then the sequence $\{m \theta\}_{m \in K}$ in $k_{\infty}$ is uniformly distributed modulo 1.

There is two nature generalizations of Theorem 1.2, and the first one is uniformly distributed modulo 1 for linear forms. Let $L(x)=L\left(x_{1}, x_{2}\right.$, $\left.\ldots, x_{s}\right)=a_{1} x_{1}+a_{2} x_{2}+\cdots+a_{s} x_{s}$, where $\alpha_{i} \in k_{\infty} . L(x)$ is said to be a linear form over $k_{\infty}$ in variables $x_{1}, x_{2}, \ldots, x_{s}$. We show that

Theorem 1.3. Let $L_{i}(x)(1 \leq i \leq n)$ be $n$ linear forms in the $s$ variables $x_{1}, x_{2}, \ldots, x_{s}$. Suppose that the only set of polynomial vectors $A=\left(A_{1}, A_{2}, \ldots, A_{n}\right) \in K^{n}$ such that

$$
A_{1} L_{1}(x)+A_{2} L_{2}(x)+\cdots+A_{n} L_{n}(x),
$$

has polynomial coefficients in $x_{1}, x_{2}, \ldots, x_{s}$ is $A=0$. Then the sequence of vectors $z^{(m)}=\left(L_{1}(m), L_{2}(m), \ldots, L_{n}(m)\right)$ for $m=\left(m_{1}, \ldots, m_{s}\right) \in K^{s}$ is uniformly distributed modulo 1 as $|m|:=\min _{1 \leq i \leq n}\left|m_{i}\right| \rightarrow \infty$.

Let $s=1$. As a straightforward consequence, we have

Corollary 1.2. Let $\theta_{1}, \theta_{2}, \ldots, \theta_{n}$ be $n$ elements in $k_{\infty}$ such that $\left\{1, \theta_{1}, \ldots, \theta_{n}\right\}$ are linearly independent over $k$. Then the sequence of vectors $z^{(m)}=\left(m \theta_{1}, m \theta_{2}, \ldots, m \theta_{n}\right)$ for $m \in K$ is uniformly distributed modulo 1. 
Next, we show that another example of uniformly distributed modulo 1 for polynomial functions.

Theorem 1.4. Let $f(x)=a_{n} x^{n}+\cdots+a_{1} x+a_{0}$ be a polynomial over $k_{\infty}$ of degree less than $p$ with the variable $x$. Suppose that $f(x)$ has at least one irrational function coefficient $a_{j}$ with $j \geq 1\left(i . e ., a_{j} \notin k\right)$. Then, the sequence $\{f(m)\}_{m \in K}$ is uniformly distributed modulo 1.

Clearly, if $n=1$, Theorem 1.4 becomes Theorem 1.2. All of the results we present here are very famous in the real number field, for details we refer the reader to ([3], Chapter 4). Throughout this paper, the notation $\alpha \equiv \beta(\bmod 1)$ means that there is a polynomial $A$ such that $\alpha=A+\beta$, where $\alpha, \beta \in k_{\infty}$. In particular, we have $\alpha=\langle\alpha\rangle(\bmod 1)$ for all $\alpha \in k_{\infty}$. This result has been proved by Carlitz in a very elementary method (see [11]) and also other scholars gave a proof in similar method. Now we will give a proof based on Fourier transformation.

\section{Proof of the Weyl's Criteria}

To prove Theorem 1.1, we need some basic techniques of harmonic analysis on $k_{\infty}$. The reader should consult ([4], Chapter 2) and ([6], Chapter 2) for details. Let $f$ be a complex valued function and $f \in L^{1}\left(k_{\infty}\right)$, the Fourier transform $\hat{f}$ takes the form

$$
\hat{f}(x)=\int_{k_{\infty}} f(t) \psi(x t) d t, \quad x \in k_{\infty}
$$

where $\psi$ is an additive character on $k_{\infty}$. If $\hat{f} \in L^{1}\left(k_{\infty}\right)$, then its Fourier transform via the conjugate of $\psi$ takes the form

$$
\hat{\hat{f}}(t)=\int_{k_{\infty}} \hat{f}(x) \psi(-t x) d x, \quad t \in k_{\infty} .
$$


Lemma 2.1. If $\hat{f} \in L^{1}\left(k_{\infty}\right)$ for continuous $f \in L^{1}\left(k_{\infty}\right)$, then

$$
\hat{\hat{f}}(t)=q^{2-n(\psi)} f(t)
$$

for all $t \in k_{\infty}$, where $n(\psi)$ is the conductor of $\psi$.

Proof. From the general theory (see [6], or page 26 of [4]), we know that $\hat{\hat{f}}=c_{\psi} f$ for some constant $c_{\psi}$ which is independent of $f$. To determine this constant, we take $f$ to be the characteristic function of $\mathfrak{P}$. It is easily seen that

$$
\hat{f}(x)=\int_{k_{\infty}} f(t) \psi(x t) d t=\int_{\mathfrak{P}} \psi(x t) d t
$$

Since $n\left(\psi_{x}\right)=n(\psi)-v(x)$ (see (1.10)), it follows that $\hat{f}$ is the characteristic function of $\mathfrak{P}_{n(\psi)-1}$. Hence by (2.2), we have

$$
\hat{\hat{f}}(t)=\int_{k_{\infty}} \hat{f}(x) \psi(-t x) d x=\int_{\mathfrak{P}_{n(\psi)-1}} \psi(-t x) d x
$$

One easily computes that

$$
\hat{\hat{f}}(t)=\mu\left(\mathfrak{P}_{n(\psi)-1}\right) f(t)=q^{2-n(\psi)} f(t) .
$$

The lemma follows at once.

Let $f$ be a continuous function on $\mathfrak{P}$. We denote by $c_{f}(A)$ the Fourier coefficients of $f$ on $\mathfrak{P}$ as follows:

$$
c_{f}(A)=\int_{\mathfrak{P}} f(t) \psi(A t) d t, \quad A \in K
$$

Since $k_{\infty}^{+}=K \times \mathfrak{P}$, we have the following Fourier series expansion. 
Lemma 2.2. Let $n(\psi)=2$, and suppose that $f$ is a continuous complex valued function on $\mathfrak{P}$ such that

$$
\sum_{A \in K}\left|c_{f}(A)\right|<\infty
$$

Then for all $t \in \mathfrak{P}$, we have

$$
f(t)=\sum_{A \in K} c_{f}(A) \psi(-A t)
$$

Proof. Extend $f$ to all of $k_{\infty}$ by defining $f(t)=0$ for $t \in k_{\infty}-\mathfrak{P}$. Since $\mathfrak{P}$ is open in $k_{\infty}$, thus $f$ is continuous on $k_{\infty}$, and

$$
\hat{f}(t)=\int_{\mathfrak{P}} f(t) \psi(x t) d t
$$

We note that if $x \in \mathfrak{P}_{i}, y \in \mathfrak{P}_{j}$, then $x y \in \mathfrak{P}_{i+j}$, in particular, we have $x y \in \mathfrak{P}_{2}$, if $x \in \mathfrak{P}$ and $y \in \mathfrak{P}$. Since $n(\psi)=2$, it follows that $\psi(x y)=1$ for all $x \in \mathfrak{P}$ and $y \in \mathfrak{P}$. We see that for $x=b+y$ with $y \in \mathfrak{P}$, then

$$
\hat{f}(x)=\hat{f}(b+y)=\int_{\mathfrak{P}} f(t) \psi(t(b+y)) d t=\int_{\mathfrak{P}} f(t) \psi(t b) d t=\hat{f}(b)
$$

Hence, $\hat{f}$ is periodic on $k_{\infty}$ with $\mathfrak{P}$ as a group of periods. By the definition of $f$ on $k_{\infty}$, we have

$$
\hat{f}(A)=\int_{k_{\infty}} f(x) \psi(A x) d x=\int_{\mathfrak{P}} f(x) \psi(A x) d x=c_{f}(A) .
$$

It follows that

$$
\begin{aligned}
\int_{k_{\infty}}|\hat{f}(x)| d t & =\sum_{A \in K} \int_{A+\mathfrak{P}}|\hat{f}(x)| d x \\
& =\sum_{A \in K}|\hat{f}(A)|=\sum_{A \in K}\left|c_{f}(A)\right|<\infty,
\end{aligned}
$$


by the assumption. Thus $\hat{f} \in L^{1}\left(k_{\infty}\right)$. If $t \in \mathfrak{P}$, by Lemma 2.1, we have

$$
\begin{aligned}
f(t) & =\int_{k_{\infty}} \hat{f}(x) \psi(-t x) d x \\
& =\sum_{A \in K} \hat{f}(A) \int_{A+\mathfrak{P}} \psi(-t x) d x \\
& =\sum_{A \in K} \hat{f}(A) \psi(-t A) \int_{\mathfrak{P}} \psi(-t x) d x \\
& =\sum_{A \in K} \hat{f}(A) \psi(-A t) .
\end{aligned}
$$

We complete the proof of Lemma 2.2.

Proof of Theorem 1.1. To simplify the notations, we assume that $s=1$, since there are no great additional complications when $s>1$. Our vectors $z^{(m)}(1 \leq m<\infty)$ are thus substantially elements in $\mathfrak{P}$, which we shall denote by $z_{m}(1 \leq m<\infty)$. To prove Theorem 1.1, it is enough to prove the cycle of implications about $z_{m}$

$$
(\mathrm{A}) \rightarrow(\mathrm{B}) \rightarrow(\mathrm{C}) \rightarrow(\mathrm{D}) \rightarrow(\mathrm{A}),
$$

where $z_{m} \in \mathfrak{P}$ for $1 \leq m<\infty$, and (A), (B), (C), and (D) are statements about $z_{m}$ as follows.

Statement (A). $z_{m}(1 \leq m<\infty)$ is uniformly distributed in $\mathfrak{P}$.

Statement (B). Suppose that $\alpha \in \mathfrak{P}$ and $r$ is a positive integer given, then

$$
M^{-1} F_{M}(\alpha, r) \rightarrow q^{1-r}, \text { as } M \rightarrow \infty
$$

where $M \geq 1$ is a positive integer, $F_{M}(\alpha, r)$ (as before) is the number of solutions of

$$
z_{m} \in \alpha+\mathfrak{P}_{r}, \quad 1 \leq m \leq M
$$


Uniformity with respect to $\alpha$ and $r$ is not assumed.

Statement (C).

$$
M^{-1} \sum_{1 \leq m \leq M} f\left(z_{m}\right) \rightarrow \int_{\mathfrak{P}} f(z) d z, \text { as } M \rightarrow \infty
$$

for all functions $f(z)$ Haar-integrable in $\mathfrak{P}$.

Statement (D). Let $\psi$ be any additive character with $n(\psi)=2$, then

$$
M^{-1} \sum_{1 \leq m \leq M} \psi\left(A z_{m}\right) \rightarrow 0, \text { as } M \rightarrow \infty,
$$

for all polynomials $A \neq 0$. Again, no uniformity with respect to $A$ is assumed.

Proof that (A) implies (B). It is trivial, since (B) is an ostensibly weaker form of $(A)$.

Proof that (B) implies (C). By considering the real and imaginary parts of $f(z)$ separately, we may suppose without loss of generality that $f(z)$ is a real valued function and, by adding an appropriate constant to $f(z)$, that $f(z) \geq 0$. Since $f$ is Haar-integrable in $\mathfrak{P}$, for each $r \in \mathbb{Z}$, $r \geq 1$, we have

$$
\int_{\mathfrak{P}} f(z) d z=\sum_{\alpha \in \mathfrak{P} / \mathfrak{P}_{r}} \int_{\alpha+\mathfrak{P}_{r}} f(t) d t
$$

Denote $\gamma_{\alpha}$ and $\Gamma_{\alpha}$ by

$$
\gamma_{\alpha}=\min _{t \in \alpha+\mathfrak{P}_{r}} f(t), \text { and } \Gamma_{\alpha}=\max _{t \in \alpha+\mathfrak{P}_{r}} f(t)
$$

For each $\epsilon>0$, if $r$ is sufficiently large, then we have

$$
\int_{\mathfrak{P}} f(z) d z-\epsilon \leq q^{1-r} \sum_{\alpha \in \mathfrak{P} / \mathfrak{P}_{r}} \gamma_{\alpha} \leq q^{1-r} \sum_{\alpha \in \mathfrak{P} / \mathfrak{P}_{r}} \Gamma_{\alpha} \leq \int_{\mathfrak{P}} f(z) d z+\epsilon .
$$


It is easy to see that

$$
M^{-1} \sum_{1 \leq m \leq M} f\left(z_{m}\right)=\sum_{\alpha \in \mathfrak{P} / \mathfrak{P} r} M^{-1} \sum_{\substack{1 \leq m \leq M \\ z_{m} \in \alpha+\mathfrak{P}_{r}}} f\left(z_{m}\right) .
$$

If $M$ is sufficiently large, by statement (B) we have

$$
(1-\epsilon) q^{1-r} \sum_{\alpha \in \mathfrak{P} / \mathfrak{P}_{r}} \gamma_{\alpha} \leq M^{-1} \sum_{1 \leq m \leq M} f\left(z_{m}\right) \leq(1+\epsilon) q^{1-r} \sum_{\alpha \in \mathfrak{P} / \mathfrak{P}_{r}} \Gamma_{\alpha} .
$$

If follows that

$$
M^{-1} \sum_{1 \leq m \leq M} f\left(z_{m}\right) \leq(1+\epsilon) q^{1-r} \sum_{\alpha \in \mathfrak{P} / \mathfrak{P} r} \Gamma_{\alpha} \leq(1+\epsilon)\left(\int_{\mathfrak{P}} f(z) d z+\epsilon\right) .
$$

On the other hand, we have similarly

$$
M^{-1} \sum_{1 \leq m \leq M} f\left(z_{m}\right) \geq(1-\epsilon) q^{1-r} \sum_{\alpha \in \mathfrak{P} / \mathfrak{P}_{r}} \gamma_{\alpha} \geq(1-\epsilon)\left(\int_{\mathfrak{P}} f(z) d z-\epsilon\right) .
$$

Therefore, we have

$$
M^{-1} \sum_{1 \leq m \leq M} f\left(z_{m}\right) \rightarrow \int_{\mathfrak{P}} f(z) d z, \text { as } M \rightarrow \infty .
$$

Proof that (C) implies (D). Since $z_{m} \in \mathfrak{P}(1 \leq m<\infty)$, we write $\psi\left(A z_{m}\right)=\psi_{A}\left(z_{m}\right)$, where $n\left(\psi_{A}\right)=n(\psi)-v(A) \geq 2$ if $A$ is a polynomial and $A \neq 0$. We see that $\psi_{A}$ is a nontrivial additive character on $\mathfrak{P}$ for all $A \in K$ and $A \neq 0$. It follows that

$$
\int_{\mathfrak{P}} \psi(A t) d t=\left\{\begin{array}{l}
1, \text { if } A=0 \\
0, \text { if } A \in K, A \neq 0
\end{array}\right.
$$

Since $\psi_{A}$ is continuous on $\mathfrak{P}$, by $(\mathrm{C})$, we have

$$
M^{-1} \sum_{1 \leq m \leq M} \psi\left(A z_{m}\right) \rightarrow \int_{\mathfrak{P}} \psi(A z) d z=0
$$

for all nonzero polynomials $A$. 
Proof that (D) implies (A). Suppose $\alpha \in \mathfrak{P}$ and $r \in \mathbb{Z}$ with $r \geq 1$. Let $\chi_{\alpha, r}$ be the characteristic function of $\alpha+\mathfrak{P}_{r}$. By the definition of $F_{M}(\alpha, r)$, we see that

$$
M^{-1} F_{M}(\alpha, r)=M^{-1} \sum_{1 \leq m \leq M} \chi_{\alpha, r}\left(z_{m}\right)
$$

To make use of Lemma 2.2, we must compute the Fourier coefficients of $\chi_{\alpha, r}$. Let $A \in K$, it is easily seen that

$$
\begin{aligned}
c_{\chi_{\alpha, r}}(A) & =\int_{\mathfrak{P}} \chi_{\alpha, r}(t) \psi(A t) d t \\
& =\int_{\alpha+\mathfrak{P}_{r}} \psi(A t) d t=\psi(A \alpha) \int_{\mathfrak{P}_{r}} \psi(A x) d x .
\end{aligned}
$$

Since $n\left(\psi_{A}\right)=2-v(A)$, we have

$$
c_{\chi_{\alpha, r}}(A)= \begin{cases}\psi(A \alpha) q^{1-r}, & \text { if }|A| \leq q^{r-2}, \\ 0, & \text { if }|A|>q^{r-2} .\end{cases}
$$

Then

$$
\sum_{A \in K}\left|c_{\chi_{\alpha, r}}(A)\right|<\infty
$$

By Lemma 2.2, the Fourier expansion of $\chi_{\alpha, r}$ on $\mathfrak{P}$ is

$$
\begin{aligned}
\chi_{\alpha, r}\left(z_{m}\right) & =\sum_{A \in K} c_{\chi_{\alpha, r}}(A) \psi\left(-A z_{m}\right) \\
& =q^{1-r} \sum_{\substack{A \in K \\
|A| \leq q^{r-2}}} \psi(A \alpha) \psi\left(-A z_{m}\right) .
\end{aligned}
$$


It follows by (2.9) that

$$
\begin{aligned}
D_{M} & =\sup _{\alpha \in \mathfrak{P}, r \geq 1}\left|q^{1-r} \sum_{\substack{A \in K \\
|A| \leq q^{r-2}}} \psi(A \alpha) M^{-1} \sum_{1 \leq m \leq M} \psi\left(-A z_{m}\right)-q^{1-r}\right| \\
& =\sup _{\alpha \in \mathfrak{P}, r \geq 1}\left|q^{1-r} \sum_{\substack{A \in K \\
0<|A| \leq q^{r-2}}} \psi(A \alpha) M^{-1} \sum_{1 \leq m \leq M} \psi\left(-A z_{m}\right)\right| \\
& \leq q \max _{\substack{A \in K \\
A \neq 0}} M^{-1}\left|\sum_{\substack{1 \leq m \leq M \\
\mid}} \psi\left(A z_{m}\right)\right| .
\end{aligned}
$$

By statement (D), we have $D_{M} \rightarrow 0$ as $M \rightarrow \infty$. This is the proof of Theorem 1.1.

Proof of Corollary 1.1. Without loss of generality, we may assume that $s=1$. Let $z_{m}(1 \leq m<\infty)$ be elements in $k_{\infty}$, not necessarily restricted to lie in $\mathfrak{P}$, we have $z_{m} \equiv\left\langle z_{m}\right\rangle(\bmod 1)$. Suppose that $\psi$ is an additive character with $n(\psi)=2$ and $\psi(\alpha)=\psi(\beta)$ if $\alpha \equiv \beta(\bmod 1)$. Then $\psi(A)=1$ for all $A \in K$, and

$$
\sum_{1 \leq m \leq M} \psi\left(A z_{m}\right)=\sum_{1 \leq m \leq M} \psi\left(A\left\langle z_{m}\right\rangle\right)
$$

The assertion of Corollary 1.1 follows by Theorem 1.1 immediately.

\section{Uniform Distribution for Linear Forms}

In this section, we first introduce an additive character $\psi$ with $n(\psi)=2$, and $\psi(\alpha)=\psi(\beta)$ if $\alpha=\beta(\bmod 1)$, so that the assertion of Corollary 1.1 makes sense. If $\alpha \in k_{\infty}$, then $\alpha$ can be uniquely written as a Laurent series as follows:

$$
\alpha=\sum_{i=s}^{+\infty} a_{i}\left(\frac{1}{T}\right)^{i}, s \in \mathbb{Z}, a_{i} \in \mathbb{F}_{q} \text {, and } a_{s} \neq 0 .
$$


For every positive integer $n \geq 1$, we set $\tau_{n}(\alpha)=a_{n}$, which is a map from $k_{\infty}$ to $\mathbb{F}_{q}$ such that $\tau_{n}(\alpha \alpha)=a \tau_{n}(\alpha)$ for all $a \in \mathbb{F}_{q}, \tau_{n}(\alpha+\beta)=$ $\tau_{n}(\alpha)+\tau_{n}(\beta)$, and $\tau_{n}(A)=0$ for all $A \in K(n \geq 1)$. In particular, we have

$$
\tau_{n}\left(T^{i} \alpha\right)=\tau_{n+i}(\alpha) \text { for all } i \geq 0,
$$

and

$$
\tau_{n}(\alpha) \neq 0 \text {, if } n=v(\alpha)
$$

Let $\lambda$ be a fixed primitive $p$-th root of 1 , we thus introduce an additive character $\psi^{(n)}$ as follows:

$$
\psi^{(n)}(\alpha)=\lambda^{\operatorname{tr}\left(\tau_{n}(\alpha)\right)}=\lambda^{\operatorname{tr}\left(a_{n}\right)}
$$

where tr is the trace map from $\mathbb{F}_{q}$ to $\mathbb{F}_{p}$. Obviously, $\psi^{(n)}$ is an additive character on $k_{\infty}$ with the conductor $n\left(\psi^{(n)}\right)=n+1$ for each positive integer $n$. In particular, we have $n\left(\psi^{(1)}\right)=2$, and

$$
\psi^{(1)}(\alpha+A)=\psi^{(1)}(\alpha), \text { for all } \alpha \in k_{\infty}, \text { and } A \in K .
$$

In the sequel of this paper, we fix $\psi=\psi^{(1)} \cdot \psi$ should play a role of exponential function in $k_{\infty}$.

Suppose that $A . H \in K$ and $A \neq 0, H \neq 0, \theta \in k_{\infty}$, we denote by $S_{A}(\theta, H)$ the exponential sums as follows:

$$
S_{A}(\theta, H)=\sum_{\substack{m \in K \\|m|<|H|}} \psi(m A \theta)
$$

where $\psi=\psi^{(1)}$ given by (3.4). 
Lemma 3.1. (i) If $\theta \in k$ is a rational function, then there is a polynomial $A \neq 0$ such that

$$
S_{A}(\theta, H)=|H|, \text { for all } H \in K \text { with } H \neq 0 .
$$

(ii) If $\theta \notin k$ is an irrational function in $k_{\infty}$ and $A \in K$ with $A \neq 0$, then we have

$$
S_{A}(\theta, H)=0, \text { if }|H| \geq\|A \theta\|^{-1},
$$

where $\|\alpha\|=|\langle\alpha\rangle|\left(\alpha \in k_{\infty}\right)$ is the smallest distance from $\alpha$ to any element of $K$ (see (1.4) above).

Proof. (i) is trivial. Since $\theta$ is a rational function, there is a polynomial $A \neq 0$ such that $A \theta \in K$. We thus have $\psi(m A \theta)=1$ for all $m \in K$ and $S_{A}(\theta, H)=|H|$ for all $H \in K$ with $H \neq 0$.

To prove (ii), we note that by (3.2)

$$
\psi^{(n)}\left(T^{i} \theta\right)=\lambda^{\operatorname{tr}\left(\tau_{n}\left(T^{i} \theta\right)\right)}=\lambda^{\operatorname{tr}\left(\tau_{n+i}(\theta)\right)}=\psi^{i+n}(\theta) .
$$

Let $\operatorname{deg}(H)=h \geq 1, \quad$ and $\quad m=b_{h-1} T^{h-1}+b_{h-2} T^{h-2}+\cdots+b_{1} T+b_{0}$. Then, we may rewrite $S_{A}(\theta, H)$ as follows

$$
S_{A}(\theta, H)=\prod_{i=0}^{h-1}\left(\sum_{b_{i} \in \mathbb{F}_{q}} \psi\left(b_{i} T^{i} A \theta\right)\right) .
$$

Since $|H| \geq\|A \theta\|^{-1}$ by assumption, we thus have $h \geq v(\langle A \theta\rangle) \geq 1$. Then there exists one of $i$ such that $0 \leq i \leq h-1$, and $i=v(\langle A \theta\rangle)-1$. Let $i+1=v(\langle A \theta\rangle)$, we have by (3.9)

$$
\begin{aligned}
\sum_{b_{i} \in \mathbb{F}_{q}} \psi\left(b_{i} T^{i} A \theta\right) & =\sum_{b_{i} \in \mathbb{F}_{q}} \psi\left(T^{i} b_{i}\langle A \theta\rangle\right)=\sum_{b_{i} \in \mathbb{F}_{q}} \psi^{1+i}\left(b_{i}\langle A \theta\rangle\right) \\
& =\sum_{b_{i} \in \mathbb{F}_{q}} \lambda^{\operatorname{tr}\left(\tau_{1+i}\left(b_{i}\langle A \theta\rangle\right)\right)}=\sum_{b_{i} \in \mathbb{F}_{q}} \lambda^{\operatorname{tr}\left(b_{i} \tau_{1+i}(\langle A \theta\rangle)\right)}=0,
\end{aligned}
$$

because of $\tau_{1+i}(\langle A \theta\rangle) \neq 0$ by (3.3). It follows that $S_{A}(\theta, H)=0$. We complete the proof of Lemma 3.1. 
As a straightforward consequence of Corollary 1.1 and Lemma 3.1, we have the following corollary, which contains the assertion of Theorem 1.2 .

Corollary 3.1. If $\theta \in k_{\infty}$ and $\theta \notin k$. then the sequence $\{m \theta\}_{m \in K}$ is uniformly distributed modulo 1 . If $\theta \in k$, then it is not uniformly distributed modulo 1.

There is a high dimensional version of Lemma 3.1. Let $L_{1}(x), \ldots, L_{n}(x)$ be $n$ linear forms in variables $x=\left(x_{1}, x_{2}, \ldots, x_{n}\right)$ given by

$$
\left[\begin{array}{c}
L_{1}(x) \\
\vdots \\
L_{n}(x)
\end{array}\right]=B\left[\begin{array}{c}
x_{1} \\
\vdots \\
x_{s}
\end{array}\right],
$$

where $B=\left(a_{i j}\right)_{n \times s}$ is an $n \times s$ matrix over $k_{\infty}$. We denote

$$
S_{A}(B, H)=\sum_{\substack{m_{1} \in K \\\left|m_{1}\right|<\left|H_{1}\right|}} \cdots \sum_{\substack{m_{s} \in K \\\left|m_{s}\right|<\left|H_{s}\right|}} \psi\left(A_{1} L_{1}(m)+\cdots+A_{n} L_{n}(m)\right)
$$

where $H=\left(H_{1}, \ldots, H_{s}\right) \in K^{s}, A=\left(A_{1}, A_{2}, \ldots, A_{n}\right) \in K^{n}$, and $m=$ $\left(m_{1}, \ldots, m_{s}\right) \in K^{s}$. We set $|H|=\min \left\{\left|H_{i}\right|: 1 \leq i \leq s\right\}$.

Lemma 3.2. If $L_{1}(x), \ldots, L_{n}(x)$ are $n$ linear forms given by (3.11), and the only set of polynomial vector $A=\left(A_{1}, \ldots, A_{n}\right) \in K^{n}$ such that $A_{1} L_{1}(x)+\cdots+A_{n} L_{n}(x)$ has polynomial coefficients in $x_{1}, x_{2}, \ldots, x_{s}$ is $A=0$. Then, we have

$$
S_{A}(B, H)=0,
$$

for sufficiently large $|H|$, where $A=\left(A_{1}, \ldots, A_{n}\right) \in K^{n}$ is a nonzero polynomial vector. 
Proof. If $A=\left(A_{1}, \ldots, A_{n}\right) \in K^{n}$ and $A \neq 0$ is given, we may write

$$
A_{1} L_{1}(x)+\cdots A_{n} L_{n}(x)=c_{1} x_{1}+c_{2} x_{2}+\cdots+c_{n} x_{n},
$$

where $c_{i} \in k_{\infty}$ depends only on $A=\left(A_{1}, \ldots, A_{n}\right)$ and the matrix $B$. Since $A \neq 0$, there is at least a $c_{i} \notin k$. By (3.12), we have

$$
S_{A}(B, H)=\prod_{j=1}^{s}\left(\sum_{\substack{m_{j} \in K \\\left|m_{j}\right|<\left|H_{j}\right|}} \psi\left(m_{j} c_{j}\right)\right) .
$$

Suppose that $c_{i} \notin k$, we thus have by Lemma 3.1,

$$
\sum_{\substack{m_{i} \in K \\\left|m_{i}\right|<\left|H_{i}\right|}} \psi\left(m_{i} c_{i}\right)=0, \text { as }\left|H_{i}\right|>\left\|c_{i}\right\|^{-1} .
$$

If follows that $S_{A}(B, H)=0$ as $|H|$ is large enough. We complete the proof of Lemma 3.2.

Now Theorem 1.3 follows immediately from Theorem 1.1 and the above lemma.

\section{Uniform Distribution for Polynomial Functions}

In this section, we give a proof of Theorem 1.4. To do this we need the following lemmas.

Lemma 4.1. Let $u(m)$ be a complex valued function on $K$, and $N, M \in K$ be two polynomials such that $1 \leq|N| \leq|M|$. Then

$$
|N|\left|\sum_{\substack{m \in K \\|m|<|M|}} u(m)\right|^{2} \leq|M|\left(\sum_{\substack{m \in K \\|m|<|M|}}|u(m)|^{2}+\sum_{\substack{h \in K \\ 0<|h|<|N|}} \sum_{\substack{m \in K \\|m|<|M|}} u(m) \overline{u(m+h)}\right) .
$$


Proof. Let $g \in K$ be a fixed polynomial such that $|g|<|N|<|M|$, it is easily seen that

$$
\sum_{\substack{m \in K \\|m|<|M|}} u(m)=\sum_{\substack{t \in K \\|t|<|M|}} u(t-g) .
$$

If follows that

$$
|N| \sum_{\substack{m \in K \\|m|<|M|}} u(m)=\sum_{\substack{t \in K \\|t|<|M|}} \sum_{\substack{g \in K \\|g|<|N|}} u(t-g) .
$$

By Schwartz's inequality, we have

$$
\begin{aligned}
& |N|^{2}\left|\sum_{\substack{m \in K \\
|m|<|M|}} u(m)\right|^{2} \leq|M| \sum_{\substack{t \in K \\
|t|<|M|}}\left|\sum_{\substack{g \in \in K \\
|g|<|N|}} u(t-g)\right|^{2} \\
& \quad=|M| \sum_{\substack{t \in K \\
|t|<|M|}} \sum_{\substack{g_{1}, g_{2} \in K \\
\left|g_{1}\right|<|N|,\left|g_{2}\right|<|N|}} u\left(t-g_{1}\right) \overline{u\left(t-g_{2}\right)} \\
& =|M||N| \sum_{\substack{m \in K \\
|m|<|M|}}|u(m)|^{2}+|M| \sum_{\substack{t \in K \\
|t|<|M|}} \sum_{g_{1} \neq g_{2}} u\left(t-g_{1}\right) \overline{u\left(t-g_{2}\right)} .
\end{aligned}
$$

Let $m=t-g_{1}$ and $h=g_{1}-g_{2}$ in the above inequality. Then the right-hand side of (4.3) becomes

$$
|N||M|\left(\sum_{\substack{m \in K \\|m|<|M|}}|u(m)|^{2}+\sum_{\substack{h \in K \\ 0<|h|<|M||m|<|<| M \mid}} u(m) \overline{u(m+h)}\right) .
$$

The lemma follows immediately. 
Lemma 4.2. Let $z_{m} \in k_{\infty}$ for $m \in K$, and suppose that

$$
|M|^{-1} \sum_{\substack{m \in K \\|m|<|M|}} \psi\left(z_{m+h}-z_{m}\right) \rightarrow 0(|M| \rightarrow \infty)
$$

for each $h \in K, h \neq 0$, not necessarily uniformly in $h$. Then

$$
|M|^{-1} \sum_{\substack{m \in K \\|m|<|M|}} \psi\left(z_{m}\right) \rightarrow 0(|M| \rightarrow \infty)
$$

Proof. Put $u(m)=\psi\left(z_{m}\right)$ in Lemma 4.1, then for all $N, M \in K$, $0<|N| \leq|M|$ we have

$$
|N|\left|\sum_{\substack{m \in K \\|m|<|M|}} \psi\left(z_{m}\right)\right|^{2} \leq|M|^{2}+|M| \sum_{\substack{h \in K \\ 0<|h|<|N|}} \sum_{\substack{m \in K \\ m \in|<| M \mid}} \psi\left(z_{m}\right) \overline{\psi\left(z_{m+h}\right)} .
$$

It follows that

$$
|M|^{-2}\left|\sum_{\substack{m \in K \\|m|<|M|}} \psi\left(z_{m}\right)\right|^{2} \leq \frac{1}{|N|}+\frac{1}{|N||M|} \sum_{\substack{h \in K \\ 0<|h|<|N|}}\left|\sum_{\substack{m \in K \\ m|<| M \mid}} \psi\left(z_{m+h}-z_{m}\right)\right| .
$$

If, now, $N$ is fixed and let $|M| \rightarrow \infty$, the right-hand side of (4.4) tends to $\frac{1}{|N|}$, which is arbitrarily small by appropriate initial choice of $N$. Hence the left-hand side of (4.4) must tends to 0 as $|M| \rightarrow \infty$. We complete the proof of Lemma 4.2 .

Lemma 4.3. A sufficient condition for the sequence $\left\{z_{m}\right\}_{m \in K}$ in $k_{\infty}$ to be uniformly distributed modulo 1 is that the sequence $\left\{z_{m+h}-z_{m}\right\}_{m \in K}$ is uniformly distributed modulo 1 for each polynomial $h \neq 0$. 
Proof. By the hypothesis and Corollary 1.1, we have

$$
|M|^{-1} \sum_{\substack{m \in K \\|m|<|M|}} \psi\left(A\left(z_{m+h}-z_{m}\right)\right) \rightarrow 0, \text { as }|M| \rightarrow \infty,
$$

for all polynomials $A$ and $h$ with $A \neq 0, h \neq 0$. By Lemma 4.2 applied to $\psi\left(A z_{m}\right)$ we deduce

$$
|M|^{-1} \sum_{\substack{m \in K \\|m|<|M|}} \psi\left(A z_{m}\right) \rightarrow 0, \text { as }|M| \rightarrow \infty,
$$

for all $A \in K, A \neq 0$. The sequence $\left\{z_{m}\right\}$ is thus uniformly distributed modulo 1 by Corollary 1.1 again.

Proof of Theorem 1.4. Since $f(x)=a_{n} x^{n}+\cdots+a_{1} x+a_{0} \in k_{\infty}[x]$ has at least one irrational function coefficient $a_{j}$ with $j \geq 1$, we suppose first that the leading coefficient $a_{n}$ is an irrational function (i.e., $a_{n} \notin k$ ). When $n=1$, the result has been proved in Theorem 1.2, we thus may assume that $n>1$, and the result has been proved for $n-1$. For any fixed polynomial $h \neq 0$, we denote that $z_{m}=f(m)$ for all $m \in K$, and thus

$$
z_{m+h}-z_{m}=f(m+h)-f(m)
$$

which is a polynomial in $m$ of degree $n-1$ with the irrational function leading coefficient $h a_{n} C_{n}^{1}$. With $\operatorname{deg} f(x)<p$. We always have $h a_{n} C_{n}^{1}$ is not zero. Hence the result for $n$ follows from that for $n-1$ and Lemma 4.3 .

If, however, $a_{n}$ is a rational function and there is some $s(1 \leq s<n)$ such that $a_{s}$ is an irrational function but $a_{s+1}, \ldots, a_{n}$ are rational functions. Let $N \in K, N \neq 0$, such that $N a_{s+1}, \ldots, N a_{n}$ are polynomials. It is clearly enough to show that

$$
\xi_{m}=f(N m+h), \quad m \in K,
$$


is uniformly distributed modulo 1 for each $h \in K$ and $|h|<|N|$. But

$$
\begin{aligned}
\xi_{m} & =a_{0}+a_{1}(N m+h)+\cdots+a_{n}(N m+h)^{n} \\
& \equiv a_{0}+a_{1}(N m+h)+\cdots+a_{s}(N m+h)^{s}+a_{s+1} h^{s+1}+\cdots+a_{n} h^{n}(\bmod 1) \\
& =b_{0}+b_{1} m+\cdots+b_{s} m^{s}
\end{aligned}
$$

where $b_{0}, b_{1}, \ldots, b_{s}$ are independent of $m$. In particular, $b_{s}=N^{s} a_{s}$ is an irrational function. This is the first case, so the theorem is proved generally.

\section{Acknowledgement}

I would like to thank Professor Jacques Peyriere for a valuable discussion about the Fourier transform of Haar integral.

\section{References}

[1] E. Bombieri and W. Gubler, Heights in Diophantine Geometry, Cambridge University Press, 2006.

DOI: https://doi.org/10.1017/CBO9780511542879

[2] Y. Bugeaud, Approximation by Algebraic Numbers, Cambridge Tracts in Mathematics 160, Cambridge University Press, 2004.

DOI: https://doi.org/10.1017/CBO9780511542886

[3] J. W. S. Cassels, Diophantine Approximation, Cambridge University Press, London, 1965.

[4] G. W. Effinger and D. R. Hayes, Additive Number Theory of Polynomials Over a Finite Field, Clarendon Press, Oxford, 1991.

[5] A. Lasjaunias, A survey of Diophantine approximation in fields of power series, Monatshefte für Mathematik 130(3) (2000), 211-229.

DOI: https://doi.org/10.1007/s006050070036

[6] M. H. Taibleson, Fourier Analysis on Local Fields, Princeton University Press and University of Tokyo Press, 1975.

[7] D. S. Thakur, Function Field Arithmetic, World Scientific, 2004.

[8] D. S. Thakur, From rationality to transcendence in finite characteristic, Journées Annuelles (2012), 21-48. 
[9] Z. Zheng, Simultaneous Diophantine Approximation in Function Fields, To appear.

[10] Z. Zheng, Simultaneous Diophantine Approximation in Function Fields (II), To appear.

[11] L. Cailitz, Diophantine approximation in fields of characteristic $p$, Transaction of the American Mathematical Society 72(2) (1952), 187-208.

DOI: https://doi.org/10.1090/S0002-9947-1952-0048503-0 